Western University

Scholarship@Western

3-1-2021

\title{
Timing of CGM initiation in pediatric diabetes: The CGM TIME
} Trial.

Margaret L Lawson

Kate C Verbeeten

Jennilea M Courtney

Brenda J Bradley

Karen McAssey

See next page for additional authors

Follow this and additional works at: https://ir.lib.uwo.ca/paedpub

Part of the Pediatrics Commons

\section{Citation of this paper:}

Lawson, Margaret L; Verbeeten, Kate C; Courtney, Jennilea M; Bradley, Brenda J; McAssey, Karen; Clarson, Cheril; Kirsch, Susan; Curtis, Jacqueline R; Mahmud, Farid H; Richardson, Christine; Cooper, Tammy; Chan, Jason; and Tang, Ken, "Timing of CGM initiation in pediatric diabetes: The CGM TIME Trial." (2021).

Paediatrics Publications. 530.

https://ir.lib.uwo.ca/paedpub/530 


\section{Authors}

Margaret L Lawson, Kate C Verbeeten, Jennilea M Courtney, Brenda J Bradley, Karen McAssey, Cheril Clarson, Susan Kirsch, Jacqueline R Curtis, Farid H Mahmud, Christine Richardson, Tammy Cooper, Jason Chan, and Ken Tang 


\title{
Timing of CGM initiation in pediatric diabetes: The CGM TIME Trial
}

\author{
Margaret L. Lawson $^{1,2}$ (1) | Kate C. Verbeeten ${ }^{1,2}$ | Jennilea M. Courtney ${ }^{2}$ | \\ Brenda J. Bradley $^{2}$ | Karen McAssey ${ }^{3}$ | Cheril Clarson ${ }^{4}$ | Susan Kirsch ${ }^{5}$ | \\ Jacqueline R Curtis $^{6}$ | Farid H Mahmud ${ }^{6}$ | Christine Richardson ${ }^{1}$ | \\ Tammy Cooper ${ }^{1}$ | Jason Chan ${ }^{2}$ | Ken Tang ${ }^{2}$
}

\author{
${ }^{1}$ Division of Endocrinology and Metabolism, \\ Department of Pediatrics, Children's Hospital \\ of Eastern Ontario, Ottawa, Ontario, Canada \\ ${ }^{2}$ Clinical Research Unit, CHEO Research \\ Institute, Ottawa, Ontario, Canada \\ ${ }^{3}$ Department of Pediatrics, McMaster \\ Children's Hospital, Hamilton, Ontario, Canada \\ ${ }^{4}$ Department of Pediatrics, Children's Hospital, \\ London Health Sciences Centre, Lawson \\ Health Research Institute, London, Ontario, \\ Canada \\ ${ }^{5}$ Department of Pediatrics, Markham- \\ Stouffville Hospital, Markham, Ontario, \\ Canada \\ ${ }^{6}$ Division of Endocrinology, Department of \\ Pediatrics, Hospital for Sick Children, Toronto, \\ Ontario, Canada \\ Correspondence \\ Margaret L. Lawson, Division of Endocrinology \\ and Metabolism, Department of Pediatrics, \\ Children's Hospital of Eastern Ontario, \\ 401 Smyth Road, Ottawa, \\ ON, Canada K1H $8 \mathrm{~L} 1$. \\ Email: lawson@cheo.on.ca \\ Funding information \\ Federal Economic Development Fund; Juvenile \\ Diabetes Research Foundation Canada, \\ Grant/Award Number: 80-2010-585
}

\begin{abstract}
Objective: To determine whether timing of CGM initiation offering low glucose suspend (LGS) affects CGM adherence in children and youth starting insulin pump therapy.

Methods: A 5-site RCT of pump-naïve subjects (aged 5-18 years) with type 1 diabetes (T1D) for at least 1 year compared simultaneous pump and CGM initiation offering LGS vs standard pump therapy with CGM initiation delayed for 6 months. Primary outcome was CGM adherence (hours per 28 days) (MiniMed ${ }^{\text {TM }}$ Paradigm $^{\text {TM }}$ Veo $^{\text {TM }}$ system; CareLink Pro ${ }^{\mathrm{TM}}$ software) over 6 months after CGM initiation. Secondary outcome HbA1c was measured centrally. Linear mixed-models and ordinary least squares models were fitted to estimate effect of intervention, and covariates baseline age, T1D duration, HbA1c, gender, ethnicity, hypoglycemia history, clinical site, and association between CGM adherence and $\mathrm{HbA1c}$.

Results: The trial randomized 144/152 (95\%) eligible subjects. Baseline mean age was $11.5 \pm 3.3(\mathrm{SD})$ years, T1D duration $3.4 \pm 3.1$ years, and $\mathrm{HbA1c} 7.9 \pm 0.9 \%$. Six months after CGM initiation, adjusted mean difference in CGM adherence was 62.4 hours per 28 days greater in the Simultaneous Group compared to Delayed Group $(P=.007)$. There was no difference in mean HbA1c at 6 months. However, for each 100 hours of CGM use per 28 -day period, $\mathrm{HbA} 1 \mathrm{c}$ was $0.39 \%(95 \% \mathrm{Cl} 0.10 \%$ $0.69 \%)$ lower. Higher CGM adherence was associated with reduced time with glucose $>10 \mathrm{mmol} / \mathrm{L}(P<.001)$.

Conclusion: CGM adherence was higher after 6 months when initiated at same time as pump therapy compared to starting CGM 6 months after pump therapy. Greater CGM adherence was associated with improved HbA1c.
\end{abstract}




\section{KEYWORDS}

adherence, continuous glucose monitoring, glycemic control, insulin pump therapy, site differences

Continuous glucose monitoring (CGM) may improve glycemic control and quality of life in individuals with type 1 diabetes. Uptake of and adherence to CGM has been studied in adults, children, and youth with type 1 diabetes with significant differences between these populations. ${ }^{1-8}$ The studies are not directly comparable due to methodological differences. A common finding in all age groups was the positive correlation between proportion of time spent using CGM and improvements in glycemic control ${ }^{1-8}$; CGM adherence was significantly lower in children and youth than adults. ${ }^{1,3,4,7}$ Low glucose suspend (LGS) feature of CGM has been demonstrated to reduce frequency of hypoglycemia without compromising safety or glycemic control. ${ }^{9,10}$ However, the effect of timing of CGM initiation with option of LGS on glycemic control has not been explored in pediatrics. We hypothesized that simultaneous initiation of CGM offering LGS and insulin pump therapy in children and youth will be associated with greater CGM adherence than their later initiation. Our study's objective was to determine whether timing of CGM initiation offering LGS, simultaneously with pump initiation or 6 months later, affects CGM adherence and/or HbA1c over the subsequent 6 months in children and youth with type 1 diabetes. The trial was performed in 2011 to 2014, when currently used sensors were not available, CGM was not commonly used before pump therapy, and time-in-range was not the standard for reporting glycemic control. We feel that our findings are still relevant and that the main results are generalizable as CGM becomes more user-friendly each year.

\section{RESEARCH DESIGN AND METHODS}

The CGM TIME Trial was a 12-month multicenter randomized controlled trial. Children and youth with type 1 diabetes for at least 1 year who were initiating insulin pump therapy were randomly assigned to start CGM offering LGS with their pump start (Simultaneous Group) or 6 months later (Delayed Group). Randomization was performed centrally, stratified by study center and by age (5-12 years vs 13-18 years), using a computer-generated randomization schedule with variable block size. Full details of the protocol have been published. ${ }^{11}$ There were no upper or lower limits for baseline HbA1c. Participants were required to meet provincial criteria for starting insulin pump therapy which include duration of diabetes ( $\geq 1$ year), glycemic control (HbA1c <10\%), and regular blood glucose monitoring and clinic visits. There was no run-in period to establish participants' acceptance and willingness to wear CGM. Informed consent, plus assent where indicated, was required. The trial was approved by the Institutional Ethics Boards of each participating site. CONSORT Standards for design and reporting of clinical trials were followed.

\section{1 | Study intervention}

Participants received the Medtronic Veo ${ }^{\mathrm{TM}}$ pump, Contour ${ }^{\circledR}$ Link BG meter, Medtronic Enlite ${ }^{\mathrm{TM}}$ sensors (under a Health Canada Investigational Testing Authorization until fully approved by Health Canada in April 2013), Minilink ${ }^{T M}$ REAL-Time transmitter, and CareLink ${ }^{T M}$ Personal software. All participants received standard diabetes care for children and youth at their site including the same insulin formulations, multidisciplinary diabetes care and education; multiple daily injections were rarely used. Diabetes nurse educators were trained to provide identical pump and CGM teaching to participants in the two randomization groups. ${ }^{12}$ Pump training included two pump training sessions, CareLink ${ }^{\mathrm{TM}}$ training, daily telephone calls for the first 10 days after pump initiation, and a telephone education session 1 month after pump start. CGM support was provided by four phone calls in the first 10 days after CGM initiation, and telephone education 1 month later. Training and programming of pump and CGM settings were standardized for all subjects, including use of saline via the pump and insulin via injections during the first week. ${ }^{11,12}$ The only difference between randomization groups was the timing of the CGM education sessions relative to the pump education sessions.

LGS feature was activated in second week of CGM use, and other CGM settings were initiated in standardized and individualized stepwise fashion developed by the Study Group. ${ }^{13}$ Participants were instructed to upload pump and CGM data every week to CareLink ${ }^{\mathrm{TM}}$ on home computers.

\section{2 | Study outcomes}

The primary study outcome was CGM adherence expressed as mean hours per 28 days for each of the six 28-day periods following initiation of CGM. CGM initiation occurred at the first study visit for the Simultaneous Group, and at the 6-month visit for the Delayed Group. When at least 21 days of complete data were available within a given 28-day block, a projected 28 day time period was calculated: (Total number of CGM hours in a 28-day period/Days of CGM usage in a 28-day period) $\times 28$ days.

The main secondary outcome was HbA1c, analyzed centrally at baseline, 6 and 12 months (Roche Diagnostics Turbidimetric Inhibition Immunoassay, utilizing the DCCT/NGSP formula, Dynacare Laboratories, Toronto, Canada). Validated questionnaires evaluating readiness for making behavior change ${ }^{14}$ and fear of hypoglycemia ${ }^{15}$ were collected for participants and their parents. A brief de novo questionnaire at the 12 month visit assessed participants' and parents' likelihood of continuing to use CGM. 
Adverse events were collected on severe hypoglycemia and severe hyperglycemia. Severe hypoglycemia was defined as an event requiring assistance from another individual to administer carbohydrate, glucagon or other resuscitative efforts due to altered consciousness, seizure or coma. Severe hyperglycemia was defined as a hyperglycemic event involving all of the following: serum ketones or large/moderate urine ketones, arterial blood $\mathrm{pH}<7.30$ or venous $\mathrm{pH}<7.24$, serum bicarbonate $<14$, and treatment in hospital.

\section{3 | Sample size and statistical analyses}

Sample size was based on the primary outcome measure CGM use in hours per 28 days (ie, 6 time periods before the 6 month visit for the Simultaneous Group and before the 12 month visit for the Delayed Group). Assuming a SD of 56.4 hours (weighted average of SDs in the 15-24 and 8-14 year age groups in two pediatric studies ${ }^{1,2}$ and allowing for a $10 \%$ dropout rate, 64 participants per group provided $80 \%$ power with a type 1 error rate at .05 to detect a difference in CGM adherence of 403.2 hours per 28 days which equates to $60 \%$ adherence which was demonstrated to be a clinically important threshold for CGM adherence in children in the landmark CGM study. ${ }^{1}$ This sample size could also detect a difference in $\mathrm{HbA1c}$ of $0.5 \%$ at 6 or 12 months with a SD of 0.93 (weighted average of SDs at 6 months from two pediatric CGM studies ${ }^{1,2}$ ). To increase power for secondary outcomes of readiness for change and fear of hypoglycemia, sample size was set at maximum of 150 .

Demographic characteristics were described by randomization group. Categorical variables were summarized in proportions, and continuous variables with normal distributions with mean and SD. Comparisons between randomization groups were tested for statistical significance using Student's $t$ test for age, duration of diabetes, and HbA1c. Chi square or Fisher's exact test were used as appropriate for the categorical variables of age group, gender, ethnicity, and history of severe hypoglycemia in the 12 months prior to study entry. The Student's $t$ test was used to examine the univariate association between mean CGM hours and randomization groups in each of the six 28-day time periods after CGM initiation. Fisher's exact test was used to compare percentages of participants not using CGM and those using CGM $>60 \%$ of the time for each randomization group in each 28-day block after CGM initiation. Chi square test was used to compare CGM satisfaction scores between randomization groups.

Generalized linear mixed modeling examined association between randomization group and CGM adherence over time. Covariates included age group, gender, ethnicity (Caucasian vs non-Caucasian), history of severe hypoglycemia in the 12 months before study entry, site, time, time ${ }^{2}$, and an interaction factor between time and randomization group. The intercept and time (the six 28-day periods) were specified as random effects while randomization group and its interaction with time were specified as fixed effects. These within-participant random effects allowed for better understanding of the variability in CGM adherence and the correlation of CGM adherence between baseline and subsequent time points. Site 1 was chosen as the reference group, as its sample size was largest. A time ${ }^{2}$ term was included to allow for nonlinearity. The estimates from the models were reported along with standard errors and $95 \%$ confidence interval.

$\mathrm{HbA1}$ cs within each randomization group were summarized with mean and SD at study entry, 6 months, and 12 months. Student's $t$ test was used to compare the mean $\mathrm{HbA} 1 \mathrm{cs}$ of the randomization groups 6 months after CGM initiation, as well as change in $\mathrm{HbA} 1 \mathrm{c}$ between study entry and 6 months after CGM initiation. Ordinary least squares regression was used to determine the effect of various parameters on HbA1c 6 months after CGM initiation; covariates included baseline $\mathrm{HbA1c}$, gender, age group, randomization group, ethnicity (Caucasian vs non-Caucasian), history of severe hypoglycemia in the 12 months before study entry, and site.

Average CGM adherence over the six time periods was used to analyze association between CGM adherence and HbA1c 6 months after CGM initiation and area under the curve for projected time spent in hypoglycemia $<3.9 \mathrm{mmol} / \mathrm{L}(70 \mathrm{mg} / \mathrm{dL})$ and hyperglycemia $>10 \mathrm{mmol} / \mathrm{L}$ (180 mg/dL). Ordinary least squares regression was used for both sets of analyses, with a restricted cubic spline technique applied to allow for non-linear effect of CGM hours. HbA1c analysis was adjusted for baseline $\mathrm{HbA1c}$, age group, gender, site, ethnicity, and history of severe hypoglycemia. The hypoglycemia and hyperglycemia analyses included cluster adjustment and adjustment for age group, gender, and site.

A P-value of less than .05 was deemed statistically significant. Statistical analysis was performed by the CHEO Research Institute using $R$ version 3.4.2. ${ }^{16}$

\section{2 | RESULTS}

\subsection{Study population}

Three hundred and fifty-three children and youth with type 1 diabetes were assessed for eligibility (CONSORT Flow Diagram, Supporting Information Figure 1), which required all participants to be naïve to pump and CGM. One hundred and fifty-two met the inclusion criteria and $94.7 \%$ consented to participation. One hundred and forty-four participants were randomized, with 73 allocated to Simultaneous Group and 71 to Delayed Group. Following randomization, 139 participants started pump therapy. There were no significant differences between groups in mean age, age category, diabetes duration, ethnicity, or history of severe hypoglycemia in the 12 months before study entry (Table 1). The Simultaneous Group had higher HbA1c at study entry $(8.05 \%+/-1.01 \%$ vs $7.72 \%+/-0.88 \%, P=.024)$ (Table 1$)$.

\subsection{Association between timing of CGM initiation and subsequent CGM adherence}

Complete information on CGM adherence in the first 6 months of CGM was available for 124 participants. CareLink ${ }^{\mathrm{TM}}$ collected incomplete CGM adherence information from 15 separate participants in 


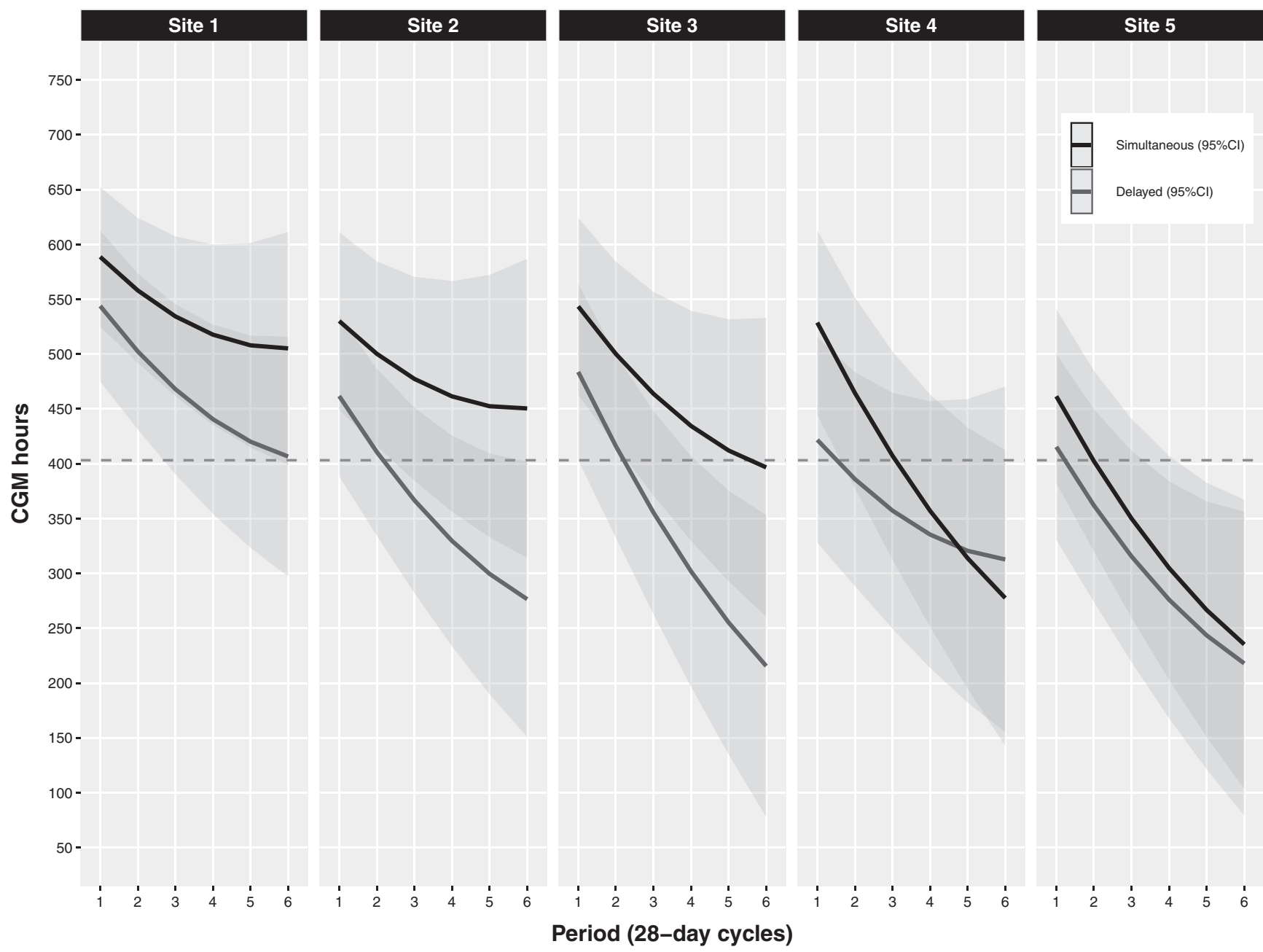

FIGURE 1 Comparison of mean CGM adherence in the Simultaneous and Delayed Groups in the six 28 day periods after the introduction of CGM, separated by (A) study site and (B) the child's gender

37 time periods. Five patient-time period values were excluded as there were less than 21 days of CareLink ${ }^{\mathrm{TM}}$ CGM adherence data in a given 28 day period; for the remaining 32 time periods, "projected CGM hours per 28 days" was calculated as described above.

Univariate analysis showed that the Simultaneous Group had consistently higher CGM adherence hours in all six time periods (Table 2). The adjusted mean difference in CGM adherence hours between randomization groups was statistically significant at all time points, and showed increasing benefit of simultaneous CGM introduction over time. The multivariate model showed that individuals in the Simultaneous Group used CGM 62.4 more hours per 28-day period ( $P=.007$ ), equivalent to an extra 2.2 hours per day, when other factors were held constant. The proportion of participants in each randomization group who wore CGM $>60 \%$ of the time (equivalent to 403.2 hours per 28-day period) decreased for both groups over time, with proportion of participants in the Simultaneous Group greater than in the Delayed Group in all time periods. The difference was statistically significant in periods 3, 4, and 5. The proportion of participants not using CGM increased over time for both randomization groups with no significant difference between the groups. Neither age group nor gender was significantly associated with CGM adherence.

There was an unexpected difference in CGM adherence among the sites (Figure 1, Table 3) with a clear separation between the Simultaneous and Delayed groups in three of the sites, favoring Simultaneous Group, while in the other two sites CGM adherence rapidly decreased in both groups. The multivariate model identified the latter two sites as having significantly lower CGM adherence compared to the reference site, with a difference of $87.4(P=.016)$ and $126.9(P<.001)$ fewer hours per 28 days (3.1 and 4.5 hours per day respectively when each site's randomization groups were combined). There were no differences in baseline demographics of participants in the five sites.

\subsection{Association between timing of CGM initiation and HbA1c 6 months after CGM initiation}

HbA1c was statistically different between randomization groups at study entry (Table 1). For this reason, post hoc it was decided that the 
TA B LE 1 Baseline Demographics of the Study Population

\begin{tabular}{|c|c|c|c|}
\hline Patient Characteristics at Study Entry & Simultaneous Group $(N=70)$ & Delayed Group $(\mathrm{N}=69)$ & $P$-value \\
\hline Age in years (mean $\pm S D$ ) & $11.49(3.34)$ & $11.47(3.35)$ & .963 \\
\hline \multicolumn{4}{|l|}{ Age category in years $(\mathrm{N}, \%)$} \\
\hline Age 13 to 18 years & $28 / 70(40.0)$ & $26 / 69(37.7)$ & \\
\hline Diabetes duration in years (mean $\pm S D$ ) & $3.55(3.14)$ & $3.25(2.95)$ & .966 \\
\hline Boys & $40 / 70(57.1)$ & $37 / 69(53.6)$ & \\
\hline $\mathrm{HbA1c}$ (mean $\pm \mathrm{SD}$ ) at study entry & & & .024 \\
\hline Percentage & $8.05(1.01)$ & $7.72(0.88)$ & \\
\hline $\mathrm{mmol} / \mathrm{mol}$ & $64.5(12.5)$ & $60.9(13.9)$ & \\
\hline Asian or Pacific Islander & $2 / 70(2.9)$ & $4 / 69(5.8)$ & \\
\hline Hispanic & 0 & 0 & \\
\hline Other & $3 / 70(4.3)$ & $6 / 69(8.7)$ & \\
\hline $\begin{array}{l}\text { Number of episodes of severe hypoglycemia in the } \\
12 \text { months prior to study entry }(\mathrm{N}, \%)\end{array}$ & $7(10)$ & $7(10)$ & .485 \\
\hline
\end{tabular}

TAB LE 2 Association between timing of CGM initiation relative to pump start and CGM adherence (expressed in hours per 28 days) over next 6 months

\begin{tabular}{|c|c|c|c|c|c|c|c|c|c|}
\hline $\begin{array}{l}\text { Months } \\
\text { after } \\
\text { CGM } \\
\text { initiation }\end{array}$ & $\begin{array}{l}\text { Randomization } \\
\text { Group }\end{array}$ & $\begin{array}{l}\mathrm{N} \\
\text { (Missing) }\end{array}$ & $\begin{array}{l}\text { Unadjusted } \\
\text { Mean CGM } \\
\text { hours per } \\
28 \text { days (SD) }\end{array}$ & $\begin{array}{l}\text { Adjusted mean } \\
\text { difference in CGM } \\
\text { adherence hours } \\
\text { between groups }^{\mathrm{a}}\end{array}$ & SE & $\begin{array}{l}95 \% \\
\text { Confidence } \\
\text { Interval (hours } \\
\text { per } 28 \text { days) }\end{array}$ & $\begin{array}{l}P \text { - } \\
\text { value }\end{array}$ & $\begin{array}{l}\text { Participants } \\
\text { using CGM } \\
>60 \% \text { of the } \\
\text { time }(\%)^{b}\end{array}$ & $\begin{array}{l}P \text { - } \\
\text { value }\end{array}$ \\
\hline 1 & Delayed & $67(1)$ & 505.1 (143.7) & & & & & 80.6 & \\
\hline 2 & Simultaneous & $70(0)$ & $502.9(166.8)$ & 67.5 & 23.6 & 21.1 to 113.8 & .004 & 80.0 & .455 \\
\hline \multirow[t]{2}{*}{3} & Simultaneous & $69(1)$ & $486.3(177.9)$ & 72.5 & 26.3 & 20.8 to 124.2 & .006 & 82.6 & .016 \\
\hline & Delayed & $67(1)$ & $408.0(221.1)$ & & & & & 62.7 & \\
\hline \multirow[t]{2}{*}{4} & Simultaneous & $70(0)$ & $463.9(199.4)$ & 77.5 & 30.3 & 18.1 to 137.0 & .011 & 72.9 & .017 \\
\hline & Delayed & $66(2)$ & 386.7 (206.4) & & & & & 51.5 & \\
\hline 6 & Delayed & $65(3)$ & 340.2 (239.4) & & & & & 50.8 & \\
\hline
\end{tabular}

${ }^{a} 60 \%$ CGM use is equivalent to 403.2 hours per 28 days.

bexpressed in hours per 28 days; the adjusted mean hours was higher in the Simultaneous Group at all time points.

outcome measure HbA1c after 6 months of CGM use would be calculated two ways. Six months after CGM initiation, mean HbA1c in the Simultaneous Group was 7.94\% (SD 0.84\%) and in the Delayed Group it was $7.94 \%$ (SD 0.91\%) $(P=.68)$. However the change in HbA1c between study entry and 6 months after CGM initiation was $-0.12 \%$ (SD $0.91 \%$ ) for Simultaneous Group and $+0.26 \%$ (SD 0.77\%) for
Delayed Group $(P=.01)$. A multivariate analysis exploring factors associated with $\mathrm{HbA1c} 6$ months after CGM initiation identified baseline HbA1c $(P<.001)$ and one of the study sites $(P=.023)$ as the only relevant parameters. In the multivariate model, randomization group was not significantly associated with HbA1c 6 months after CGM initiation. 
TAB LE 3 Association between CGM adherence (expressed in hours per 28 days), randomization group, study site and parameters in mixed effects model

\begin{tabular}{|c|c|c|c|c|c|}
\hline Parameter & Coefficient & SE & $\begin{array}{l}\text { 95\% Confidence Interval } \\
\text { (hours per } 28 \text { days) }\end{array}$ & $\mathbf{t}$ & $P$-value \\
\hline Randomization Group (Simultaneous) & 62.4 & 22.6 & 17.8 to 107.1 & 2.8 & .007 \\
\hline Gender (female) & 39.8 & 23.1 & -5.8 to 85.4 & 1.7 & .086 \\
\hline Site 2 & -69.7 & 33.4 & -135.9 to -3.6 & -2.1 & .039 \\
\hline Site 5 & -126.9 & 33.6 & -193.3 to -60.4 & -3.8 & $<.001$ \\
\hline Time & -52.9 & 8.1 & -68.7 to -37.1 & -6.6 & $<.001$ \\
\hline Time $^{2}$ & 3.5 & 1.3 & 1.0 to 6.1 & 2.7 & .007 \\
\hline Simultaneous group $\times$ time & 0.6 & 34.8 & -68.2 to 69.5 & 0.0 & .411 \\
\hline
\end{tabular}

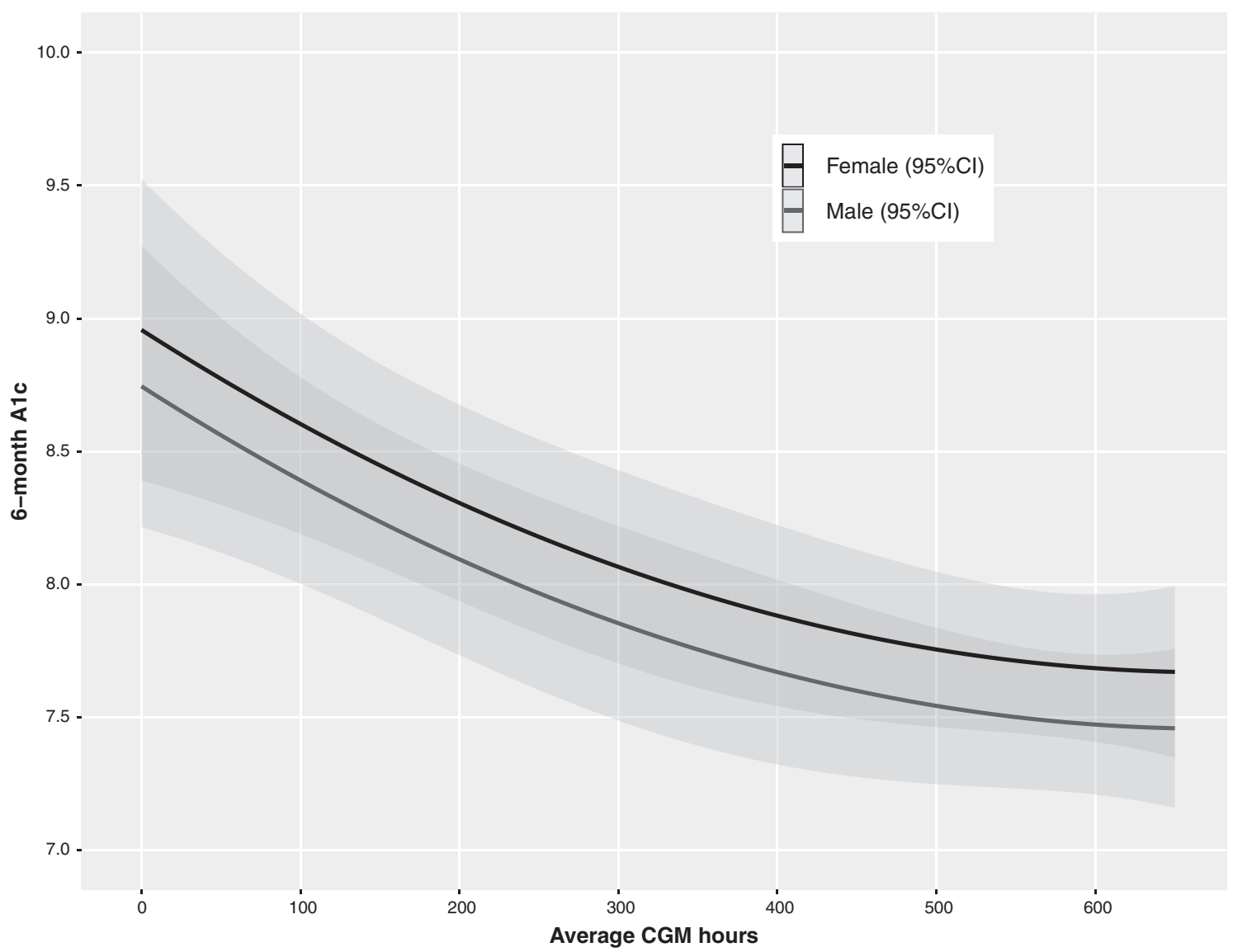

FIGURE 2 Association between CGM adherence hours per 28 days and HbA1C 6 months after CGM initiation as determined by ordinary least squares regression

\subsection{Exploratory analyses}

A significant negative association was found between average CGM adherence hours and HbA1c 6 months after CGM initiation (Figure 2,
Supporting Information Table 1), such that every additional 100 hours of CGM use per 28-day period (3.57 hours per day) was associated with a $0.38 \%$ (95\% confidence interval $0.09-0.68 \%$ ) reduction in $\operatorname{HbA1c}(P=.013)$. 
A model investigating estimated area under the curve for proportion of time spent with blood glucose $<3.9 \mathrm{mmol} / \mathrm{L}(70 \mathrm{mg} / \mathrm{dL})$ and average CGM adherence hours did not find a significant association between these two factors ( $P=.192$ ), however study site was found to be associated with estimated time spent in hypoglycemia $(P<.001)$. In contrast, increasing CGM use was significantly associated with reduction in time spent with blood glucose $>10 \mathrm{mmol} / \mathrm{L}$ $(180 \mathrm{mg} / \mathrm{dL})(P<.001)$. Study site was also associated with time spent in hyperglycemia $(P=.003$ ) (Supporting Information Figure $2 A, B$ ).

\subsection{Adverse events}

There were four episodes of severe hypoglycemia in each randomization group during the 12 months of the study. In the Simultaneous group, three of the four participants were using CGM with LGS active at the time of the event. In the Delayed Group, two of the four participants were using CGM with LGS active at the time of the event. There were three episodes of DKA during the trial, and all occurred in participants in the Delayed Group who were not using CGM at the time.

\section{6 | Patient satisfaction}

At the conclusion of the trial, participants were asked to rate on a 5-point Likert scale how likely they would be to continue CGM. $44.4 \%$ of children/youth, $64.0 \%$ of mothers, and $65.5 \%$ of fathers reported they would be "likely" or "very likely" to continue CGM. There was no statistical difference in response distribution between randomization groups.

\section{3 | DISCUSSION}

Our main conclusions were the following: first, that simultaneous initiation of CGM with the option of LGS and insulin pump therapy was associated with greater CGM adherence in the first 6 months compared to initiation of CGM with option of LGS 6 months after starting pump therapy (though this effect varied between sites); second, that there was no significant difference in mean HbA1c 6 months after CGM initiation although there was a significant difference in change in $\mathrm{HbA1c}$ favoring the Simultaneous Group; and third, that greater CGM adherence was associated with improved glycemic control at 6 months.

Our first conclusion is consistent with our hypothesis that simultaneous initiation of CGM and pump therapy would result in improved CGM adherence. This hypothesis was based on the idea that simultaneous initiation would capitalize on readiness for change that individuals in the process of changing their insulin delivery method would be experiencing, while also reinforcing the concept that CGM is an integral part of pump therapy, as opposed to an optional addition. The readiness for change hypothesis was examined by having all parents and youth who were age 10 years and older complete the validated SOCRATES questionnaire ${ }^{14}$ at baseline and 6 months later, and while motivational stage was associated with glycemic control at trial entry, it did not predict future diabetes-related behavior or HbA1c. Our CGM adherence results are supported by Moreno-Fernandez et al's 26 week study ${ }^{17}$ involving 22 adults with type 1 diabetes about to start pump therapy. Their participants were randomized to start CGM 3 weeks before starting pump therapy or 3 weeks after. Those who started CGM before pump therapy had $84.6 \%$ CGM adherence compared with $64.0 \%$ in those who started pump therapy first. Hypoglycemia, measured by CGM and by number of hypoglycemia events, was significantly lower in their early CGM group. Their study did not include the LGS option. It is surprising that in our study, CGM that offered LGS did not have an effect on frequency of hypoglycemia measured as $A U C<4 \mathrm{mmol} / \mathrm{L}$ or on frequency of severe hypoglycemia, with four episodes over 6 months in each group. This was lower than in the 12 months before the study, during which seven participants in each group reported an episode of severe hypoglycemia. The lack of effect on frequency or severity of hypoglycemia is likely because it was designed to be a pragmatic study with no minimum A1c for participation and therefore many participants entered the study with excellent control. As a result, there was a floor effect, which would have impacted results in our study. CGM offering LGS was found to be associated with lower fear of hypoglycemia in participants and their parents as measured by the Hypoglycemia Fear Scare. ${ }^{18}$

CGM adherence is known to be lower in children and youth compared to adults with type 1 diabetes. ${ }^{1,3,4,7}$ Mean CGM adherence in our study was similar to what has been reported previously in pediatric diabetes. ${ }^{1-3}$ There was a trend toward greater CGM adherence in female participants, which did not reach statistical significance $(P=.086)$. In our trial, at the 6-month mark, $65.7 \%$ of participants in the Simultaneous group and $50.8 \%$ of those in the Delayed group were using CGM over $60 \%$ of the time. This represented a significant decrease in adherence compared to the first month of CGM use for both randomization groups, though the decrease was less in the Simultaneous group. Previous studies have examined barriers to CGM adherence in adolescents, which include body image, loss of freedom, pain, annoyance with alarms, and data overload. ${ }^{19,20}$ The addition of CGM to the diabetes regimen may affect the child-parent relationship, especially if parents and children have different goals and expectations ${ }^{19}$ and may add to the already-significant burden of managing diabetes on a day-to-day basis. It makes sense that introducing CGM and pump therapy together might decrease the perceived burden of CGM by combining it with the increased flexibility that pump therapy offers to youth.

One of the strengths of the CGM TIME Trial is the structured, stepwise, and specific CGM teaching approach, most of which we described in detail in a separate publication. ${ }^{13}$ Even with this degree of standardization, differences in personal approach and institutional culture introduce variability, and this may have contributed to the unexpected difference in intervention effect between study sites. The trial was conducted when sensor technology was relatively new. 
Three of the sites had considerable experience with the Medtronic Pump CGM System prior to the study. In contrast, before the study, the other two sites had rarely or never taught and supported children and youth on the Medtronic Pump CGM System. An Australian study, ${ }^{21}$ piloted with two diabetes educators in Canada, examined diabetes educators' perceptions of factors that support and limit the use of diabetes technologies such as CGM in people with T1D, and found, while care was well intentioned, it was often not delivered with appropriate technology expertise. This demonstrates the importance of comprehensive training and support for clinic staff on diabetes technologies that are new to them so that they can teach and fully support patients and families on how to effectively use emerging technologies.

Our second and third conclusions relate to effect of CGM on $\mathrm{HbA} 1 \mathrm{c}$ and the association between CGM adherence and glycemic control. A 2011 meta-analysis ${ }^{6}$ suggested that for every 1 day per week of CGM use, HbA1c decreases by $0.15 \%$, with greater effect seen in individuals with a higher $\mathrm{HbA1c}$ before starting CGM. We did not find an effect on mean HbA1c at 6 months in either group; however, there was a significant difference between the groups in change in HbA1c 6 months after CGM initiation. While individuals in the Delayed Group had a small increase in HbA1c 6 months after CGM initiation, those in the Simultaneous Group had a small decrease. This may be related to the often-observed phenomenon of a temporary drop in $\mathrm{HbA1c}$ immediately after pump initiation, with a gradual increase over time. $^{20}$ In the Simultaneous Group, we could not separate the pump effect from the CGM effect. Mean HbA1c at study entry was $8.05 \%$ in the Simultaneous Group and 7.72\% in the Delayed Group, representing average control for children and youth, although above recommended levels. The level of metabolic control at study entry likely contributed to the lack of effect of CGM on mean $\mathrm{HbA1c}$. In agreement with the meta-analysis, we found that improvements in $\mathrm{HbA1c}$ were associated with greater CGM adherence. Area under the curve for both hypoglycemia (<3.9 mmol/L; $70 \mathrm{mg} / \mathrm{dL}$ ) and hyperglycemia ( $>10 \mathrm{mmol} / \mathrm{L}$; $180 \mathrm{mg} / \mathrm{dL}$ ) is frequently reported in pediatric CGM studies. In our study, greater adherence with CGM was associated with less hyperglycemia, a finding also reported in the SWITCH trial. $^{3}$

The main limitation of our study is the delay in publication and our inability to calculate time-in-range which in 2019 was recommended as a clinical target and outcome measurement that complements HbA1c. ${ }^{23}$ Our trial used the Enlite ${ }^{\mathrm{TM}}$ sensor and Minimed ${ }^{\mathrm{TM}}$ Paradigm $^{\mathrm{TM}} \mathrm{VeO}^{\mathrm{TM}}$ pump, however, we feel that issues of CGM adherence are likely generalizable among different CGM brands. The issues of data overload, alarm fatigue, and the interplay with normal child development and youth-parent relationships are ubiquitous. ${ }^{19}$ Adjustment for multiple comparisons was not done as generalized linear mixed modeling and ordinary least squares regression included multiple covariates including baseline $\mathrm{A} 1 \mathrm{c}$, gender and age.

Strengths of our study include good internal validity, as the only difference between groups was the timing of CGM initiation. There was also good external validity due to the intention-to-treat analysis, the low dropout rate, and minimal preselection of participants. There was no run-in period to choose participants who were most likely to succeed, unlike many previous CGM trials. ${ }^{1,3,5}$ The between-site differences reflect the reality of managing a condition that depends on the interaction of patients and the medical team.

In conclusion, our data suggest that initiating CGM with the option of LGS in a pediatric population with type 1 diabetes at the time of pump initiation is preferable to afterwards. CGM devices that have been approved since our study was completed are being started successfully before pump therapy and with multiple daily injections. ${ }^{24}$ Our results, as well as those from previous studies, notably the STAR3 trial, $^{2}$ demonstrate that young patients (and their parents) are able to adapt to both new technologies at once, and that the simultaneous use of both devices has the potential to improve diabetes management.

\section{ACKNOWLEDGEMENTS}

This trial was an oral presentation at the American Diabetes Association 78th Annual Scientific Sessions on June 24, 2018 and presented in part at the Canadian Pediatric Endocrine Group Annual Scientific Meeting on February 24, 2019. The authors acknowledge the funding provided by JDRF Canada and the Federal Economic Development Agency for Southern Ontario (FedDev Ontario) through the JDRF Canadian Clinical Trial Network (JDRF CCTN). The role of the JDRF CCTN was to conduct the peer review process prior to funds being awarded, establish statement of work agreements and the steering committee to oversee the network studies, and ensure quality assurance was maintained through audit of expenditures and monitoring by a third party contract research organization (Robarts Clinical Trials Inc.). The network has primary responsibility for oversight of presentations and publications. Funding for all authors was provided through the JDRF CCTN. The JDRF Canadian Clinical Trial Network (CCTN) is a public-private partnership including JDRF International, JDRF-Canada and the Federal Economic Development Agency for Southern Ontario, and is supported by JDRF \#80-2010-585.

\section{CONFLICT OF INTEREST}

This is an investigator-initiated trial. Pumps and CGM supplies for participants were purchased by the Study Group from Medtronic Canada at a discounted price. MLL has been a speaker, without honorarium, at educational events sponsored by Medtronic and Animas with travel reimbursement to attend these events. KCV received funding from the Canadian Pediatric Endocrine Group Fellowship Program, the Children's Hospital Academic Medical Organization, and JDRF Canada. $\mathrm{CC}$ has been a speaker with honorarium at educational events sponsored by Medtronic and Dexcom. KM and SEK have been speakers with honorarium at educational events sponsored by Medtronic, Dexcom, and Animas. For the other authors, no competing financial interests exist.

\section{AUTHOR CONTRIBUTIONS}

Margaret L. Lawson designed and coordinated the study, wrote and edited the manuscript. Kate $\mathrm{C}$. Verbeeten contributed to writing the manuscript. Ken Tang and Jason Chan performed the statistical analysis. All authors reviewed and edited the manuscript. A complete listing 
of members of the CGM TIME Trial Study Group can be found in the Online Supporting Information. Margaret L. Lawson is the guarantor of this work and takes responsibility for the accuracy of data collection and analysis.

\section{ETHICAL STATEMENT}

Ethics approval from the Institutional Ethics Boards of each participating site.

\section{PEER REVIEW}

The peer review history for this article is available at https://publons. com/publon/10.1111/pedi.13144.

\section{ORCID}

Margaret L. Lawson (D) https://orcid.org/0000-0002-2370-9552

\section{REFERENCES}

1. The Juvenile Diabetes Research Foundation Continuous Glucose Monitoring Study Group. Continuous glucose monitoring and intensive treatment of type 1 diabetes. N Engl J Med. 2008;359:1464-1476.

2. Bergenstal RM, Tamborlane WV, Ahmann A, et al. Effectiveness of sensor-augmented insulin-pump therapy in type 1 diabetes. $N$ Engl J Med. 2010;363(4):311-320.

3. Battelino T, Conget I, Olsen B, et al. The use and efficacy of continuous glucose monitoring in type 1 diabetes treated with insulin pump therapy: a randomised controlled trial. Diabetologia. 2012;55:31553162.

4. Raccah D, Sulmont V, Reznik Y, et al. Incremental value of continuous glucose monitoring when starting pump therapy in patients with poorly controlled type 1 diabetes: the RealTrend study. Diabetes Care. 2009;32(12):2245-2250.

5. Mauras N, Beck R, Xing D, et al. A randomized clinical trial to assess the efficacy and safety of real-time continuous glucose monitoring in the management of type 1 diabetes in young children aged 4 to $<10$ years. Diabetes Care. 2012; 35(2):204-10.

6. Pickup J, Freeman S, Sutton A. Glycaemic control in type 1 diabetes during real time continuous glucose monitoring compared with self monitoring of blood glucose: meta-analysis of randomised controlled trials using individual patient data. BMJ. 2011;343:d3805.

7. JDRF Continuous Glucose Monitoring Study Group. Factors predictive of use and of benefit from continuous glucose monitoring in type 1 diabetes. Diabetes Care. 2009;32(11):1947-1953.

8. Hirsch I, Abelseth J, Bode B, et al. Sensor-augmented insulin pump therapy: results of the first randomized treat-to-target study. Diabetes Technol Ther. 2008;10(5):377-383.

9. Bergenstal RM, Knoff DC, Garg SK, et al. Threshold-based insulinpump based interruption for reduction of hypoglycemia. N Engl J Med. 2013;369:224-232.

10. Weiss R, Gard SK, Bode BW, et al. Hypoglycemia reduction and changes in hemoglobin A1c in the ASPITE in-home study. Diabettes Technol Ther. 2015;17(8):542-547.

11. Lawson ML, Bradley B, McAssey K, Clarson C, Kirsch SE, Mahmud FH. The JDRF CCTN CGM TIME trial: timing of initiation of continuous glucose monitoring in established pediatric type 1 diabetes: study protocol, recruitment and baseline characteristics. BMC Pediatr. 2014;14(1):183.
12. Evans K, Richardson C, Landry A, Muileboom J, Cormack L, Lawson ML. Experience with the Enlite sensor in a multicenter pediatric study. Diabetes Educ. 2015;41(1):31-37.

13. Richardson C, Muileboom J, Evans K, Landry A, Cormack C, Lawson ML, the CGM TIME Trial Study Group and JDRF Canadian Clinical Trial Network (JDRF CCTN 1101). Successful Development and Implementation of a Standardized Approach to Initiating Continuous Glucose Monitoring in a Multicentre Pediatric Trial. Poster Presentation at the International Society for Pediatric and Adolescent Diabetes (ISPAD), Toronto, Canada, September 2014.

14. Trigwell $P$, Grant $P$, House A. Motivation and glycemic control in diabetes mellitus. J Psychosom Res. 1997;43(3):307-315.

15. Cox DJ, Irvine A, Gonder-Frederick L, Nowacek G, Butterfield J. Fear of hypoglycemia: quantification, validation, and utilization. Diabetes Care. 1987;10(5):617-621.

16. R Core Team R: A Language and Environment for Statistical Computing. Vienna, Austria: R Foundation for Statistical Computing.

17. Moreno-Fernandez J, Gomez F, Galvez Moreno M, Castano J. Clinical efficacy of two different methods to initiate sensor-augmented insulin pumps: a randomized controlled trial. J Diabetes Res. 2016;2016: 1-6.

18. Verbeeten KC, Perez Trejo ME, Tang K, Chan J et al. Fear of Hypoglyemia in children with established type 1 diabetes and their parents: effect of continuous glucose monitoring and pump therapy with option of low glucose suspend in the CGM TIME trial. Accepted for publication by Pediatric Diabetes, October 29, 2020.

19. Rashotte J, Tousignant K, Richardson C, et al. Living with sensoraugmented pump therapy in type 1 diabetes: adolescents' and parents' search for harmony. Can J Diabetes. 2014;38:256-262.

20. Patton S, Clements M. Psychological reactions associated with continuous glucose monitoring in youth. J Diabetes Sci Technol. 2016;10 (3):656-661.

21. James S, Perry L, Gallagher R, Lowe J. Diabetes Eduators: perceived experiences, supports, and barriers to use of common diabetesrelated technologies. J Diabetes Sci Tehnol. 2016;10(5):1115-1121.

22. Carlsson B, Attvall $S$, Clements $M$, et al. Insulin pump: long-term effects on glycemic control:an observational study at 10 diabetes clinics in Sweden. Diabetes Technol Ther. 2013;15(4):302-307.

23. Battelino T, Danne T, Bergenstal RM, et al. Clinical targets for continuous glucose monitoring data interpretation: recommendations from the international consensus on time in range. Diabetes Care. 2019;42 (8):1593-1603.

24. Chiang JL, Maahs DM, Garvey KC, et al. Type 1 diabetes in children and adolescents: a position statement by the American Diabetes Association. Diabetes Care. 2018;41(9):2026-2044.

\section{SUPPORTING INFORMATION}

Additional supporting information may be found online in the Supporting Information section at the end of this article.

How to cite this article: Lawson ML, Verbeeten KC, Courtney JM, et al. Timing of CGM initiation in pediatric diabetes: The CGM TIME Trial. Pediatr Diabetes. 2021;22: 279-287. https://doi.org/10.1111/pedi.13144 